\title{
Competition between noise and coupling in the induction of synchronisation
}

\author{
D. García-Álvarez ${ }^{1,2}$ (a), A. Bahraminasab ${ }^{1}$, A. Stefanovska ${ }^{1}$ and P. V. E. McClintock ${ }^{1}$ \\ 1 Department of Physics, Lancaster University - Lancaster LA1 4YB, United Kingdom \\ 2 Present address: Department of Earth and Environmental Sciences, The Open University - \\ Milton Keynes MK' 6AA, United Kingdom
}

PACS $05.45 . \mathrm{Xt}$ - Synchronisation; coupled oscillators
PACS $05.10 . \mathrm{Gg}$ - Stochastic analysis methods (Fokker-Planck, Langevin, etc.)
PACS $05.40 . \mathrm{Ca}$ - Noise

\begin{abstract}
We apply a Fokker-Planck analysis to investigate the relative influences of coupling strength and noise on the synchronisation of two phase oscillators. We go beyond earlier studies of noise-induced synchronisation (without couplings) and coupling-induced synchronisation (without common noise) to consider both effects together, and we obtain a result that is very different from a straightforward superposition of the effects of each agent acting alone: two regimes are possible depending on which agent is inducing the synchronisation. In each regime, one agent induces and the other hinders the synchronisation. In particular we show that, counter-intuitively, coupling can sometimes inhibit synchronisation.
\end{abstract}

Synchronisation occurs universally between interacting oscillatory processes both in nature and technology [1]. The phenomenon consists of the mutual adjustment of the oscillatory rhythms. It can usefully be described in terms of phase dynamics provided that the inter-oscillator interactions and noise are relatively weak [2]. Where the synchronisation is due to phase-coupling via the interoscillator interactions, the underlying mechanism is well understood. Synchronisation can also arise in a different way, however, through the influence of common noise on the two systems. This latter mechanism has been observed in many natural and experimental systems, such as lasers [3], neurons [4,5], and ecological systems [6]. Such noiseinduced synchronisation in phase oscillators has been studied by analyses of the Lyapunov exponent [7], and by use of the Fokker-Planck equation [8] to study the phenomenon in completely uncoupled phase oscillators [9-11]. In the real world, however, it is common for oscillatory systems to be influenced, not only by noise, but also by their mutual couplings [12].

In this Letter we report a systematic analysis taking simultaneous account of both possible routes to synchronisation. For added realism, we also include the effect of non-common (independent) noise. We show analytically

\footnotetext{
(a) E-mail: david.dga@gmail.com
}

and numerically how coupling and common noise compete in achieving synchronisation. We study the global synchronisation of the system via the Fokker-Planck approach, analysing the distinct roles of non-common noise, common noise, and couplings, and we reveal the competition that occurs between couplings and common noise. The most striking result is that, although coupling and common noise each favour synchronisation on their own, they can each inhibit the synchronisation induced by the other when they coexist. For example, in regimes where common-noise synchronisation dominates, stronger couplings between the subsystems implies poorer synchronisation, contrary to intuition and common belief.

The system. - We consider two coupled oscillators subject to both common and independent noises:

$$
\begin{aligned}
& \dot{x}_{\alpha}(t)=F_{\alpha}\left(x_{\alpha}(t)\right)+\epsilon_{\alpha} V_{\alpha}\left(x_{1}(t), x_{2}(t)\right) \\
& +G_{\alpha}\left(x_{\alpha}(t)\right) \sqrt{D_{\alpha}} \xi(t)+H_{\alpha}\left(x_{\alpha}(t)\right) \sqrt{E_{\alpha}} \eta_{\alpha}(t),(1)
\end{aligned}
$$

for $\alpha=1,2$. Here $x_{\alpha}$ represents the $\alpha$-th oscillator, $F_{\alpha}$ is its individual dynamics, and the $V \mathrm{~s}$ and $\epsilon \mathrm{S}$ are respectively the coupling functions and their magnitudes. $\xi(t)$ and $\eta_{\alpha}(t)$ are the common and non-common noises. $G$ and $H$ represent the couplings of the oscillators to the noises. $\xi(t)$ and $\eta_{\alpha}(t)$ are assumed to be independent, Gaussian, white noises of zero mean and unit in- 
tensity: $\langle\xi(t) \xi(s)\rangle=\delta(t-s),\left\langle\eta_{\alpha}(t) \eta_{\beta}(s)\right\rangle=\delta_{\alpha \beta} \delta(t-s)$, $\left\langle\xi(t) \eta_{\alpha}(s)\right\rangle=0$.

For weak couplings and noise intensities we can apply the well-known phase reduction method $[2,10,11]$ to get the equations for the phases:

$$
\begin{gathered}
\dot{\phi}_{\alpha}(t)=\omega_{\alpha}+\epsilon_{\alpha} v_{\alpha}\left(\phi_{1}(t), \phi_{2}(t)\right) \\
+\quad \sqrt{D_{\alpha}} \xi(t) g_{\alpha}\left(\phi_{\alpha}(t)\right)+\sqrt{E_{\alpha}} \eta_{\alpha}(t) h_{\alpha}\left(\phi_{\alpha}(t)\right) .
\end{gathered}
$$

Here $\omega_{\alpha}$ is the natural frequency of the $\alpha$-th oscillator; $v_{\alpha}$, $g_{\alpha}$ and $h_{\alpha}$ are $V_{\alpha} \partial_{x_{\alpha}} \phi_{\alpha}, G_{\alpha} \partial_{x_{\alpha}} \phi_{\alpha}$, and $H_{\alpha} \partial_{x_{\alpha}} \phi_{\alpha}$ respectively. For the general equations (1), the noises may be either multiplicative, or additive by making the functions $G$ and/or $H$ equal to unity; but the noises will become multiplicative for the phase dynamics. We seek to establish how much synchronisation of a given order $n: m[1]$ eqs. (2) can yield, quantified by evaluating how close to a constant value the generalized phase difference $\phi_{-}$stays over time. So we make an invertible change of variables from $\phi_{1}$ and $\phi_{2}$ to $\phi_{+}$and $\phi_{-}$:

$$
\begin{aligned}
\phi_{-}=m \phi_{1}-n \phi_{2}, & \phi_{+}=m \phi_{1}+n \phi_{2}, \\
\phi_{1}=\left(\phi_{+}+\phi_{-}\right) /(2 m), & \phi_{2}=\left(\phi_{+}-\phi_{-}\right) /(2 n) .
\end{aligned}
$$

The functions $v_{\alpha}, g_{\alpha}$ and $h_{\alpha}$ in (2) must be $2 \pi$-periodic in all the phases, and can therefore be written as Fourier series. As long as $\phi_{-}$stays close to a constant, it is a good approximation for the coupling function just to consider the harmonics that depend only on $\phi_{-}$, because those with any dependence on $\phi_{+}$correspond to fast oscillations [1]. We take for simplicity the lowest-order of such harmonics (which is typically the most intense): $v_{1}\left(\phi_{1}(t), \phi_{2}(t)\right)$ is therefore replaced by $a_{1} \sin \phi_{-}$, and $v_{2}$ is replaced by $-a_{2} \sin \phi_{-}$(with this notation, same signs of $a_{1} \epsilon_{1}$ and $a_{2} \epsilon_{2}$ means that the two coupling terms add up to an increased effective coupling intensity, whereas opposite signs make the two terms subtract into a weaker effective coupling, as we will see in a moment). With this approximation for the coupling function, and expanding $g_{\alpha}$ and $h_{\alpha}$ in Fourier series, the equations for the phases are

$$
\begin{aligned}
\dot{\phi}_{1}= & \omega_{1}+\varepsilon_{1} \sin \phi_{-}+\sqrt{D_{1}} \xi(t) \sum_{k} b_{1, k} \sin \left(k \phi_{1}+\theta_{1, k}\right) \\
& +\sqrt{E_{1}} \eta_{1}(t) \sum_{l} c_{1, l} \sin \left(l \phi_{1}+\vartheta_{1, l}\right) \\
\dot{\phi}_{2}= & \omega_{2}-\varepsilon_{2} \sin \phi_{-}+\sqrt{D_{2}} \xi(t) \sum_{k^{\prime}} b_{2, k^{\prime}} \sin \left(k^{\prime} \phi_{2}+\theta_{2, k^{\prime}}\right) \\
& +\sqrt{E_{2}} \eta_{2}(t) \sum_{l^{\prime}} c_{2, l^{\prime}} \sin \left(l^{\prime} \phi_{2}+\vartheta_{2, l^{\prime}}\right)
\end{aligned}
$$

where $\varepsilon_{\alpha}=a_{\alpha} \epsilon_{\alpha}$. The sums run over the positive integers (and there are no terms independent of the phase). Using these and the first equation of (3), we get

$$
\begin{aligned}
\dot{\phi}_{-}= & \omega_{-}+\varepsilon_{+} \sin \phi_{-}+\xi(t)\left[m \sum_{k} A_{1, k} \sin \left(k \phi_{1}+\theta_{1, k}\right)\right. \\
& \left.-n \sum_{k^{\prime}} A_{2, k^{\prime}} \sin \left(k^{\prime} \phi_{2}+\theta_{2, k^{\prime}}\right)\right]
\end{aligned}
$$

$$
\begin{aligned}
& +m \eta_{1}(t) \sum_{l} B_{1, l} \sin \left(l \phi_{1}+\vartheta_{1, l}\right) \\
& -n \eta_{2}(t) \sum_{l^{\prime}} B_{2, l^{\prime}} \sin \left(l^{\prime} \phi_{2}+\vartheta_{2, l^{\prime}}\right) \\
& A_{\alpha, k}=b_{\alpha, k} \sqrt{D_{\alpha}}, \quad B_{\alpha, k}=c_{\alpha, k} \sqrt{E_{\alpha}} .
\end{aligned}
$$

$\omega_{-}=m \omega_{1}-n \omega_{2}$ is the mismatch, and the "effective coupling" $\varepsilon_{+}$is defined as $m \varepsilon_{1}+n \varepsilon_{2}$. We will assume $\varepsilon_{+}>0$, so the tendency of the coupling is to drive the two oscillators at around $\phi_{-}=\pi$, as we will see later. The latter point should be noted carefully by readers accustomed to considering synchronisation near $\phi_{-}=0$, in which case $\varepsilon_{+}>0$ would be regarded as repulsive coupling. This is not our case: here, synchronisation can happen near different values of $\phi_{-}$, depending on the values of the parameters of the system (see below). We recall that, when synchronisation in our system is due to coupling, $\phi_{-}$stays around $\pi$.

Fokker-Planck analysis. - We choose to use the Ito version of the associated Fokker-Planck equation though, in fact (see below), the Stratonovich version leads to the same final results. Out of $\phi_{+}(t)$ and $\phi_{-}(t)$, the former is fast-varying: in the absence of couplings and noises, $\phi_{\alpha}(t)=\omega_{\alpha} t$, so $\phi_{+}(t)=\omega_{+} t$. For our case with couplings and noises, corrections must be made to this expression; but, provided all the couplings and noise strengths are small compared to the frequency $\omega_{\alpha}$, such corrections will be small compared to the quickly growing term $\omega_{+} t$. Thus we can take $\phi_{+}(t) \approx \omega_{+} t$, and we may integrate out $\phi_{+}$over one period in the Fokker-Planck equation. Hence only those terms that do not contain any derivative with respect to $\phi_{+}$will be relevant. The equation for the probability density $W\left(\phi_{+}, \phi_{-}, t\right)$ is [8]:

$$
\begin{aligned}
\partial_{t} W= & -\partial_{\phi_{-}}\left[\left(\omega_{-}+\varepsilon_{+} \sin \phi_{-}\right) W\right] \\
& +(1 / 2) \partial_{\phi_{-}}^{2}\left\{\left[m \sum_{k} A_{1, k} \sin \left(k \phi_{1}+\theta_{1, k}\right)\right.\right. \\
& \left.-n \sum_{k^{\prime}} A_{2, k^{\prime}} \sin \left(k^{\prime} \phi_{2}+\theta_{2, k^{\prime}}\right)\right]^{2} W \\
& +\left[m \sum_{l} B_{1, l} \sin \left(l \phi_{1}+\vartheta_{1, l}\right)\right]^{2} W \\
& \left.+\left[n \sum_{l^{\prime}} B_{2, l^{\prime}} \sin \left(l^{\prime} \phi_{2}+\vartheta_{2, l^{\prime}}\right)\right]^{2} W\right\}+\cdots,
\end{aligned}
$$

where the suspension points stand for the terms involving at least a derivative with respect to $\phi_{+}$. We will study cases where the intensity of at least one noise is enough to yield a stationary solution for which $\partial W / \partial t=0$. Then we can suppose that the probability density $W$ is almost independent of the fast variable $\phi_{+}$, because $\phi_{+} \approx \omega_{+} t$, so $t \approx \phi_{+} / \omega_{+}$and therefore $0=\partial W / \partial t \approx \omega_{+} \partial W / \partial \phi_{+}$, from which we get $\partial W / \partial \phi_{+} \approx 0$. As a result, we can work with the probability density $P\left(\phi_{-}\right)$resulting from the integration of $W$ within a period of $\phi_{+}$. From the second line of (3), we see that the system is $4 n m \pi$-periodic 
in the variables $\phi_{+}$and $\phi_{-} . P\left(\phi_{-}\right)$is then defined as $P\left(\phi_{-}\right)=(1 / 4 n m \pi) \int_{0}^{4 n m \pi} W\left(\phi_{+}, \phi_{-}\right) \mathrm{d} \phi_{+} \approx W\left(\phi_{-}\right)$, where the time dependence was taken out of $W$ because of the stationary condition, and $W$ was approximated as being independent of $\phi_{+}$in the last step.

In (5), we replace $W$ by $P\left(\phi_{-}\right)$, write $\phi_{1}$ and $\phi_{2}$ as a function of $\phi_{+}$and $\phi_{-}$, and integrate out $\phi_{+}$between 0 and $4 n m \pi$. The integrals for the terms with $A \mathrm{~s}$ and $B \mathrm{~s}$ are independent of $\phi_{-}$except for some of the cross terms coming from the first square bracket in (5): most of the latter integrate out to 0 , except for the resonant ones: i.e. those where $k=u m$ and $k^{\prime}=u n$, with $u$ integer. These resonant terms play a vital role, as we will see: it is so important that we can make a very convenient simplification preserving all the interesting features: we zero all the $A_{1, k}$ except for $k=m$, and all the $A_{2, k^{\prime}}$ except for $k^{\prime}=n$. We therefore redefine $A_{1, m} \equiv A_{1}$ and $A_{2, n} \equiv$ $A_{2}$. The $B$ s do not generate any interesting cross-terms, so we can simplify by zeroing them all except for the lowestorder terms: $B_{1,1} \equiv B_{1}$ and $B_{2,1} \equiv B_{2}$. Also for the sake of simplicity, we make 0 the two $\theta$ s and the two $\vartheta_{\mathrm{s}}$ corresponding to the former non-zero terms. We end up with a system that is simple enough to be analytically tackable, and complete enough to retain all the relevant features. After integrating out $\phi_{+}$in (5), we get:

$$
\begin{aligned}
& \frac{\partial}{\partial \phi_{-}}\left\{-\left(\omega_{-}+\varepsilon_{+} \sin \phi_{-}\right) P+\frac{1}{4} \frac{\partial}{\partial \phi_{-}}\left[\left(m^{2}\left(A_{1}^{2}+B_{1}^{2}\right)\right.\right.\right. \\
& \left.\left.\left.+n^{2}\left(A_{2}^{2}+B_{2}^{2}\right)-2 n m A_{1} A_{2} \cos \phi_{-}\right) P\right]\right\}=0
\end{aligned}
$$

Note that the integrals of the terms involving at least a derivative with respect to $\phi_{+}$are equal to zero because of periodicity. Derivations from the Ito and Stratonovich schemes here merge: had we used Stratonovich calculus, we would have had extra terms for the Fokker-Planck equation for $W\left(\phi_{+}, \phi_{-}, t\right)$, but the resulting equation for $P\left(\phi_{-}\right)$after integration over $\phi_{+}$would be identical with eq. (6). So the rest of the discussion is true for Ito as well as Stratonovich stochastic schemes. Eq. (6) shows that the expression inside the top-level bracket on the lefthand side is a constant: the probability current $S$. If we call $f_{1}\left(\phi_{-}\right)=-4\left(\omega_{-}+\varepsilon_{+} \sin \phi_{-}\right)$and $f_{2}\left(\phi_{-}\right)=m^{2}\left(A_{1}^{2}+\right.$ $\left.B_{1}^{2}\right)+n^{2}\left(A_{2}^{2}+B_{2}^{2}\right)-2 n m A_{1} A_{2} \cos \phi_{-}$, equation (6) yields $P^{\prime}\left(\phi_{-}\right)+\left[f_{1}\left(\phi_{-}\right)+f_{2}{ }^{\prime}\left(\phi_{-}\right)\right] P\left(\phi_{-}\right) / f_{2}\left(\phi_{-}\right)=S / f_{2}\left(\phi_{-}\right)$, and the general solution of the differential equation is

$$
P\left(\phi_{-}\right)=\frac{S \int_{\phi_{0}}^{\phi_{-}} \exp [V(x)] \mathrm{d} x+N}{f_{2}\left(\phi_{-}\right) \exp \left[V\left(\phi_{-}\right)\right]},
$$

where $V(x)=\int_{x_{0}}^{x} f_{1} / f_{2}$. The lower limits $\phi_{0}$ and $x_{0}$ for the former integrals are arbitrary, but once such values are chosen, they have to be kept the same. For convenience, we set both equal to $-\pi$. The constants $N$ and $S$ in (7) are obtained by requiring, first, that $P\left(\phi_{-}\right)$be $2 \pi$ periodic (because the equation to solve (6) is $2 \pi$-periodic in $\left.\phi_{-}\right)$. Secondly for normalization, we require the integral of $P\left(\phi_{-}\right)$over one period to be equal to 1 .

Note that $f_{1}(x)$ and $f_{2}(x)$ are $2 \pi$-periodic. A primitive $\widetilde{V}(x)$ of $f_{1} / f_{2}$ can be obtained analytically, valid inside the interval $-\pi \leq x \leq \pi$ :

$$
\begin{aligned}
\widetilde{V}(x)= & -\frac{4 \pi \omega_{-}}{\sqrt{R}}-\frac{8 \omega_{-}}{\sqrt{R}} \arctan \left[\frac{T \tan (x / 2)}{\sqrt{R}}\right] \\
& +\frac{2 \varepsilon_{+}}{A_{1} A_{2} m n}\left\{\ln T-\ln \left[\left(A_{1}^{2}+B_{1}^{2}\right) m^{2}\right.\right. \\
& \left.\left.+\left(A_{2}^{2}+B_{2}^{2}\right) n^{2}-2 A_{1} A_{2} m n \cos x\right]\right\}
\end{aligned}
$$

where

$$
\begin{aligned}
R= & \left(B_{1}^{2} m^{2}+B_{2}^{2} n^{2}\right)\left[\left(2 A_{1}^{2}+B_{1}^{2}\right) m^{2}\right. \\
& \left.+\left(2 A_{2}^{2}+B_{2}^{2}\right) n^{2}\right]+\left(A_{1}^{2} m^{2}-A_{2}^{2} n^{2}\right)^{2}
\end{aligned}
$$

and

$$
T=\left(A_{1} m+A_{2} n\right)^{2}+B_{1}^{2} m^{2}+B_{2}^{2} n^{2} .
$$

Also, the definite integral $I_{V}$ of $f_{1} / f_{2}$ within one period is $\widetilde{V}(\pi)-\widetilde{V}(-\pi)$

$$
I_{V}=-\frac{8 \pi \omega_{-}}{\sqrt{R}}
$$

Putting everything together, the integral that defines $V(x)$ can be obtained for any $x$, by writing $x=(2 \kappa-1) \pi+\varphi$, with $\kappa$ integer and $0 \leq \varphi \leq 2 \pi$ : then $V(x)=\kappa I_{V}+$ $\widetilde{V}(-\pi+\varphi)-\widetilde{V}(-\pi)=\kappa I_{V}+\widetilde{V}(-\pi+\varphi)$. Because $I_{V}$ is defined as the integral over one period (or from the former expression), we find

$$
V(x+2 \alpha \pi)=V(x)+\alpha I_{V}, \quad \alpha \in \mathbb{Z} .
$$

A relationship between the constants $N$ and $S$ in (7) can be obtained by imposing the periodicity condition. By requiring that $P\left(\phi_{-}+2 \pi\right)$ and $P\left(\phi_{-}\right)$be equal, we obtain a relationship between $S$ and $N: S=\left[\exp \left(I_{V}\right)-1\right] N / I_{P}$, where

$$
I_{P}=\int_{-\pi}^{\pi} \exp [V(x)] \mathrm{d} x
$$

to be obtained numerically. Note that, for $I_{V}=0$, or equivalently, the mismatch $\omega_{-}$equal to 0 , the probability current $S$ vanishes. Writing $S$ as a function of $N$ in (7):

$$
P\left(\phi_{-}\right)=\frac{N}{f_{2}\left(\phi_{-}\right) \mathrm{e}^{V\left(\phi_{-}\right)}}\left[1+\frac{\mathrm{e}^{I_{V}}-1}{I_{P}} \int_{-\pi}^{\phi_{-}} \mathrm{e}^{V(x)} \mathrm{d} x\right],
$$

where the constant $N$ normalizes $P\left(\phi_{-}\right)$within one period, $\int_{-\pi}^{\pi} P\left(\phi_{-}\right) \mathrm{d} \phi_{-}=1$.

Results for small frequency mismatch. - Some analytical results can be derived for the case of zero frequency mismatch; they also hold, at least approximately, as long as the mismatch remains small. For $\omega_{-}=0$, the 

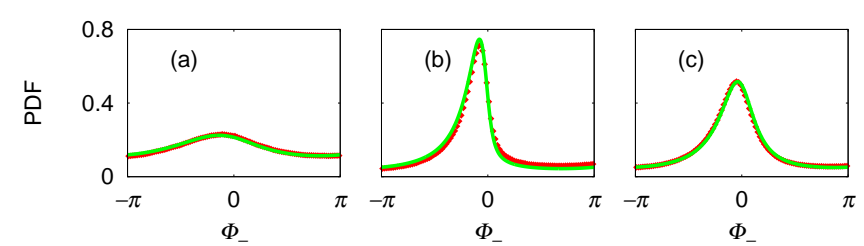

Fig. 1: Influence on synchronisation of the ratio between $A_{1}$ and $A_{2}$. (a) $A_{1}=0.1$; (b) 0.45 ; (c) 0.8 . The lines represent the theoretical probability density function from (14), with the last integral and the normalization constant $N$ obtained numerically; the points are from simulation. The plots are for $\nu_{1}=1.025, \nu_{2}=0.23, n=9, m=2, \varepsilon_{1}=0.001, \varepsilon_{2}=0.0002$, $B_{1}=0.1, B_{2}=0.02$ and $A_{2}=0.1(\nu=\omega /(2 \pi))$. All figures in this Letter correspond to a small, but non-zero, frequency mismatch.

expression for the probability density function simplifies to:

$$
\begin{aligned}
P_{0}\left(\phi_{-}\right) & =N_{1}\left[m^{2} B_{1}^{2}+n^{2} B_{2}^{2}+\left(m A_{1}-n A_{2}\right)^{2}\right. \\
& \left.+4 n m A_{1} A_{2} \sin ^{2} \frac{\phi_{-}}{2}\right]^{\left(2 \varepsilon_{+} / n m A_{1} A_{2}\right)-1},
\end{aligned}
$$

where $N_{1}$ is a constant, independent of $\phi_{-}$, to be adjusted such that the probability density be normalized to 1. Stronger synchronisation will occur when the term depending on $\phi_{-}$(last one inside the bracket) dominates the $\phi_{-}$-independent terms: the first three terms inside the bracket should be as close to zero as possible in order to obtain stronger synchronisation. Two conclusions can be drawn. First, the bigger the absolute values of $B_{1}$ and $B_{2}$, the weaker the synchronisation: independent noise is always harmful to synchronisation. Secondly, the smaller $\left|m A_{1}-n A_{2}\right|$ is, the closer the third term will be to zero. Thus the magnitudes of the couplings of the common noise to the two oscillators should be in the same $n: m$ synchronisation ratio in order to optimally enhance the strength of synchronisation. See fig. 1: for (b), $m A_{1}-n A_{2}$ is equal to 0 , and the corresponding probability density is the most localised. So we assume below that $A_{1}$ and $A_{2}$ have the same sign. For all numerical results we discretised eqs. (4) and used the Heun scheme [13] with a step of 0.01. The results of the simulations are independent of the initial conditions as we have discarded the first transient to allow enough time for the system to fall into the stationary solution - the only exception is fig. 2(a) where the noises intensities are extremely weak, as indicated in the caption.

Having derived the above optimization requirement for the ratio of $A_{1}$ and $A_{2}$, we now explore the influence of their values. From the exponent in (15) we can study the competition between coupling and common-noise. When the "effective coupling" $\varepsilon_{+}$is big compared to $A_{1} A_{2}$, more specifically $\varepsilon_{+}>n m A_{1} A_{2} / 2$, the exponent is positive, so the probability density will have its maximum at $\phi_{-}=\pi$. If the product $A_{1} A_{2}$ increases, the bracket in (15) will depend more strongly on $\phi_{-}$thereby tending to enhance syn-

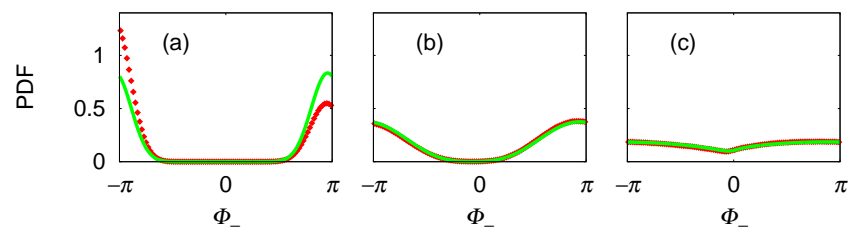

Fig. 2: Influence of the intensities of common noise on couplinginduced synchronisation. Here $\nu_{1}=1.025, \nu_{2}=0.23, n=9$, $m=2, \varepsilon_{1}=0.3, \varepsilon_{2}=0.05, B_{1}=0.1, B_{2}=0.02$, and: (a) $A_{1}=0.225$ and $A_{2}=0.05$; (b) 0.45 and 0.1 ; (c) 0.675 and 0.15. Note that $m A_{1}-n A_{2}=0$ in all three plots. For the simulations corresponding to (a), the system stays within a small interval of $\phi_{-}$: due to the weakness of the noises, it does not attain the stationary solution. The discrepancy between theory and simulation in (a) is thus attributable to the finite length of the time series.

chronisation. However, the exponent will be smaller, thus flattening the probability density and tending to hinder synchronisation. As (15) is more sensitive to the second effect than to the first, we conclude that, in the case of coupling-induced synchronisation, larger noise intensities (either common or non-common) hinder synchronisation, as is evident in fig. 2 .

When $\varepsilon_{+}<n m A_{1} A_{2} / 2$, we enter a different regime: common-noise-induced synchronisation. Because the exponent in (15) is negative, the probability density will have a maximum at $\phi_{-}=0$. In contrast to the previous case, large values of $A_{1} A_{2}$ are now beneficial in that: the $\phi_{-}$dependent term inside the bracket then dominates over the other terms; and they increase the absolute value of the exponent (we still assume $\varepsilon_{+}>0$ ): see fig. 3 ; also, compare figs. 1(c) and 1(a). We can have very narrow peaks in the probability density, and correspondingly strong synchronisation, for small values of the non-commmon noises, together with strong couplings of the common noise to the oscillators, whose "generalized difference" $\left|m A_{1}-n A_{2}\right|$ is then small (but $A_{1}$ or $A_{2}$ cannot be arbitrarily large because the approximation $\phi_{+} \approx \omega_{+} t$ would then fail; furthermore, the phase dynamics approach is valid only for weak couplings and noise). In this regime of commonnoise-induced synchronisation, a bigger value of the effective coupling $\varepsilon_{+}$will result in an exponent that is smaller in absolute value: smaller values for the couplings between the oscillators are better for common-noise-induced synchronisation, as shown in fig. 4. The origin of this competition between coupling and common noise is that each effect tends to push the system into synchronising at a different value of $\phi_{-}$. Common noise locks the system at around $\phi_{-}=0$, and the coupling term $\varepsilon_{+} \sin \phi_{-}$pushes the system away from $\phi_{-}=0$. In general, we must expect there to be competition between coupling and common noise; the exception is the very special case where both agents tend to push the system into synchronising near same value of $\phi_{-}$. 

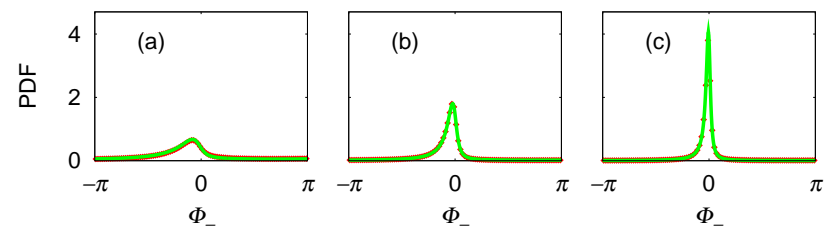

Fig. 3: Synchronisation increases with the intensity of common noise, when it is induced by the latter instead of by couplings. Here $\nu_{1}=1.025, \nu_{2}=0.23, n=9, m=2, \varepsilon_{1}=0.01, \varepsilon_{2}=$ $0.002, B_{1}=0.1, B_{2}=0.02$, and: (a) $A_{1}=0.45$ and $A_{2}=0.1$; (b) 0.9 and 0.2 ; (c) 1.8 and 0.4 . Note that $m A_{1}-n A_{2}=0$ in all three plots.

Conclusions. - We have studied phase synchronisation between two oscillators in the general case, encompassing both couplings and noise. There are two different routes to synchronisation: either via coupling or via common noise. Where one mechanism induces synchronisation, the other hinders it, corresponding to the existence of competition between them. In principle, this result allows us to distinguish coupling- and commonnoise-induced synchronisation: e.g. in the cardiorespiratory interaction, stronger noise yields weaker synchronisation [14], so that the route to synchronisation here is apparently via couplings. A striking consequence of this competition is that, for the common noise scenario, an increase in coupling inhibits synchronisation: here, the effective coupling should ideally be as small as possible.

$$
* * *
$$

We acknowledge valuable discussions with $\mathrm{T}$. Stankovski. The work was supported by the EU Project BRACCIA.

\section{REFERENCES}

[1] Pikovsky A., Rosenblum M. and Kurths J., Synchronisation. A Universal Concept in Nonlinear Sciences (Cambridge University Press) 2001.

[2] Kuramoto Y., Chemical Oscillations, Waves, and Turbulence (Springer-Verlag) 1984 .

[3] Uchida A., McAllister R. and Roy R., Phys. Rev. Lett., 93 (2004) 244102.

[4] Mainen Z. F. and Sejnowski T. J., Science, 268 (1995) 1503.

[5] Teramae J. N. and Fukai T., Phys. Rev. Lett., 101 (2008) 248105.

[6] Grenfell B. T. et al., Nature (London), 394 (1998) 674.

[7] Teramae J. N. and Tanaka D., Phys. Rev. Lett., 93 (2004) 204103.

[8] Risken H., The Fokker-Planck Equation (Springer-Verlag) 1989 .

[9] Goldobin D. S. and Pikovsky A., Phys. Rev. E, 71 (2005) 045201(R).

[10] Nakao H., Arai K., and Kawamura Y., Phys. Rev. Lett., 98 (2007) 184101.
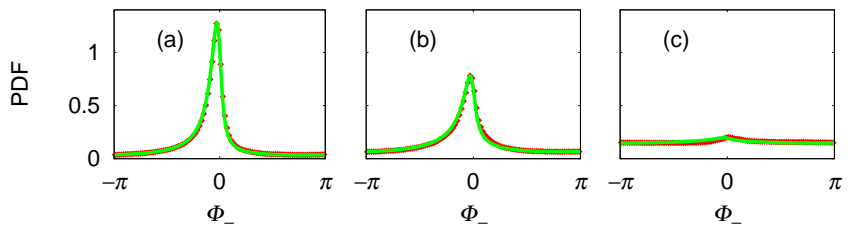

Fig. 4: Synchronisation decreases with the intensity of the effective coupling, when it is induced by common noise. Here $\nu_{1}=1.025, \nu_{2}=0.23, n=9, m=2, A_{1}=0.9, A_{2}=0.2$, $B_{1}=0.1, B_{2}=0.02$, and: (a) $\varepsilon_{1}=0.1$ and $\varepsilon_{2}=0.02$; (b) 0.2 and 0.04 ; (c) 0.4 and 0.08 .

[11] Yoshimura K. and Arai K., Phys. Rev. Lett., 101 (2008) 154101.

[12] Kenwright D. A. et al., Eur. Phys. J. B, 65 (2008) 425.

[13] Mannella R., IJMPC, 13 (2002) 1177.

[14] Stefanovska A. et al., Phys. Rev. Lett., 85 (2000) 4831. 